\title{
Post-Endoscopy Self-Limited Unspecified Fever. Rare or Common Adverse Event after Endoscopy? An Observational, Cross-Sectional Study
}

Boris Tufegdzic ( $\nabla$ drb.tufegdzic@gmail.com )

Cleveland Clinic Abu Dhabi

\section{Guus Jansen}

Cleveland Clinic Abu Dhabi

Lina Hussein

Cleveland Clinic Abu Dhabi

Valsala Visno

Cleveland Clinic Abu Dhabi

Terrence Lee-St John

Cleveland Clinic Abu Dhabi

Massimo Lamperti

Cleveland Clinic Abu Dhabi

\section{Research Article}

Keywords: Post endoscopy fever, drug-induced fever, bacteria translocation.

Posted Date: February 2nd, 2022

DOI: https://doi.org/10.21203/rs.3.rs-1275685/v1

License: (9) (i) This work is licensed under a Creative Commons Attribution 4.0 International License.

Read Full License 


\section{Abstract}

Introduction: Although post-endoscopy fever (PEF) without colon perforation or haemorrhage is believed to be rare, incidence, risk factors and the causes in the adult population have not been fully investigated. Endogenous immune response, bacteria translocation, contaminated endoscopes, or drug-induced fever are all protentional reasons that can lead to elevated temperature. The purpose of the present study was to investigate the incidence of PEF and identify the risk factors associated with the development of PEF and its outcomes.

Material and Methods: Over a three-month period, 1054 non-hospitalised patients who had an endoscopic procedure at Cleveland Clinic Abu Dhabi received a post-procedure phone call within the first 24 hours. After identifying patients with fever and obtaining verbal consent, patients were enrolled in the study using a standardised telephone interview.

Results: Thirty-four patients with PEF were identified. The highest temperature measured was 39.8 degrees Celsius. The most common single procedure performed was oesophagogastroduodenoscopy (41.2\%). Colonoscopy was performed as a single procedure in $17.6 \%$ and double procedure (oesophagogastroduodenoscopy and colonoscopy) in $41.2 \%$ of patients. Polypectomy was performed in only 5 patients (14.7\%). Multiple biopsies were performed in 26 of the 34 patients (76.5\%). Propofol was used in all endoscopy procedures and the dose was directly related to the duration of the procedure. Logistic regression revealed that no significant group differences across procedure types existed in terms of adjusted odds of fever. However, results also indicated that age has a significant negative relationship with fever - higher age is associated with lower odds of fever $(b=-0.033, p=0.024)$.

Conclusion: PEF is an unpleasant side effect and it is associated with patient discomfort, dissatisfaction and fear during the post-endoscopy recovery. Although our findings do not fully explain the possible mechanisms underlying post-endoscopy fever, this study data should increase awareness about PEF as a common side effect related to endoscopy.

\section{Introduction}

Gastrointestinal (GI) endoscopy is a common procedure performed for the evaluation and treatment of various GI tract disorders. Unfortunately, the increased use of these procedures for diagnostic and therapeutic reasons has been accompanied by a corresponding increase in the incidence of postendoscopy complications.

In our clinical practice, we have encountered patients who experienced fever after the endoscopy procedure without peritoneal signs or definitive fever foci. As a result, patients were often referred to the emergency department or admitted to the hospital to receive other unanticipated evaluations for further assessment. 
Fever, also known as pyrexia and febrile response, is defined as a temperature above the normal range due to an increase in the body's temperature set point [1]. Among adults, the average body temperature ranges from $97^{\circ}$ Fahrenheit $\left(36.1^{\circ}\right.$ Celsius) to $99^{\circ}$ Fahrenheit $\left(37.2^{\circ}\right.$ Celsius), for most people, and it is considered as the baseline normal value. A fever rarely comes without other symptoms and it is often accompanied by specific complaints like chills, fatigue, joint or muscle ache among others.

Although PEF without colon perforation or haemorrhage is believed to be rare, its incidence and risk factors in the adult population have not been investigated completely. PEF is a relatively common adverse event following endoscopy in children, with a greater risk following interventional procedures, causing concern for perforation and/or significant infection as a complication from endoscopy [2, 3]. In a study published by Kramer and colleagues [3], fever represented $21.6 \%$ of all perioperative events with an incidence of $0.55 \%$ across all pediatric endoscopic procedures [4]. Similar results are published by Coser and colleagues for adult population where fever represented $21.5 \%$ of all post-colonoscopy emergency department visits [5].

The reasons for PEF have not yet been fully investigated. Endogenous immune response, bacteria translocation, contaminated endoscopes, or drug-induced fever are all potential reasons that can lead to post-procedural elevated temperature.

The purpose of the present study was to investigate the incidence of PEF and possibly identify the risk factors associated with the development of PEF and its outcomes.

\section{Material And Methods}

Authors performed a single-center, observational, prospective study. Ethics approval for this study was provided by the Institutional Review Board (IRB) of Cleveland Clinic, Abu Dhabi, UAE, under Study Registration No. A-2019-046, on 9th September 2019.

Over a three-month period, 1054 non-hospitalized patients who had an endoscopic outpatient procedure at Cleveland Clinic Abu Dhabi were interviewed by telephone within the first 24 hours after the procedure as routine post-procedure practice. Endoscopic outpatient procedure was defined as any endoscopic procedure in which the purpose was to inspect the mucosa and/or obtain biopsies for histologic analysis or minor procedures that will not require hospitalisation and will be performed under monitored anaesthesia care (e.g. Endoscopic ultrasound (EUS), Banding, Dilatation). After identifying 34 patients with fever and obtaining verbal consent, we enrolled all 34 patients in the study performing standardized telephone interviews. The standardized telephone interview consisted of five questions related to the presence of fever and accompanying symptoms, the values obtained during the measurement, the use of antipyretics and antibiotics, as well as visits to doctors or emergency departments. Data from the remaining 1020 patients with no fever were retained and served as the comparison group in inferential analyses. Individual consent for the remaining 1020 patients with no fever for retrospective data analysis was waived by same study registration number. 
All endoscopic procedures were performed in the Department of Gastroenterology, using standard gastroscopes (GIF-H190 or GIF-HQ190; Olympus Optical Co., Ltd., Tokyo, Japan), and adult or pediatric colonoscopes (CF-HQ190 or PCF-H190; Olympus Optical Co., Ltd., Tokyo, Japan). The choice of endoscope was selected at the discretion of the proceduralist.

Monitored anaesthesia care (MAC) was used in all patients. Sedation for the procedures was provided by propofol (by continuous infusion or intermittent bolus doses). Standard American Society of Anesthesiologists recommendation monitoring (electrocardiography, pulse oximetry, non-invasive blood pressure monitoring and continuous monitoring of end-tidal CO2) were applied.

This study followed the "Strengthening the Reporting of Observational Studies in Epidemiology (STROBE)" statement guidelines for observational studies [6].

\section{Statistical Methodology}

Statistical data are presented through descriptive statistics. Discrete variables are presented as absolute number (n) and percentage (\%) and normally distributed data are shown as mean with standard deviation (SD).

Unadjusted group comparisons were made using independent samples t-test and Fisher's exact tests for continuous and categorical variables respectively. Adjusted endoscopy group differences (colonoscopy, oesophagogastroduodenoscopy, oesophagogastroduodenoscopy \& colonoscopy) in terms of odds of fever were calculated and compared using logistic regression. Group odds of fever were adjusted via inclusion of the following covariates: age (years), BMI, endoscopy duration (minutes), and propofol (mg).

All analyses were performed using Microsoft R Open 4.0.2.

\section{Results}

Demographic and clinical data of all included patients were collected and documented from electronic medical records. Patients' confidentiality was protected by assigning a de-identified patient code.

After evaluating 1054 non-hospitalized patients who had an endoscopic procedure, thirty-four patients with PEF were identified. Flow chart of study process is presented in Fig. 1. Demographic information on sex, age, ASA status, BMI and type of endoscopy procedure are presented in Table 1. The frequency of fever was similarly present in both sexes (Male-44\%: Female-56\%), with a slightly higher percentage in the female population without statistical significance. Frequency by age was more prevalent in the younger adult population (Mean [SD]-39.2[11.5]). 
Table 1

Patient demographics and sample description (ASA - American Society of Anesthesiologists physical status classification; BMI- Body Mass Index; EUS-Endoscopic ultrasound).

\begin{tabular}{|c|c|c|c|c|}
\hline & & Fever & w/o Fever & p \\
\hline Total No. Patients & & 34 & 1020 & \\
\hline Age - Mean (SD) & & $39.2(11.5)$ & $45.2(14.4)$ & .016 \\
\hline BMI - Mean (SD) & & $28.4(6.4)$ & $29(6.5)$ & .548 \\
\hline Sex - No (\%) & Male: & $15(44.1 \%)$ & $491(48.1 \%)$ & .728 \\
\hline & Female: & $19(55.9 \%)$ & $529(51.9 \%)$ & \\
\hline ASA - No (\%) & I & $4(11.8 \%)$ & & \\
\hline & II & $24(70.6 \%)$ & & \\
\hline & III & $6(17.6 \%)$ & & \\
\hline Esophagogastrodu & & $14(41.2 \%)$ & $306(30 \%)$ & .212 \\
\hline Colonoscopy - No ( & & $6(17.6 \%)$ & $305(29.9 \%)$ & \\
\hline Esophagogastrodu & lonoscopy - No (\%) & $14(41.2 \%)$ & 409 (40.1\%) & \\
\hline Intervention (EUS, & - No (\%) & $2(5.9 \%)$ & $23(2.25 \%)$ & .171 \\
\hline
\end{tabular}

Logistic regression revealed that no significant group differences existed in terms of adjusted odds of fever. However, results also indicated that age has a significant negative relationship with fever - higher age is associated with lower odds of fever $(b=-0.033, p=0.024)$. Table 2 . Our results are in contrast with previous published study published by Coser and colleagues where they did not find statistically significant relation between age and fever [5]. 
Table 2

Logistic Regression Results (BMI- Body Mass Index)

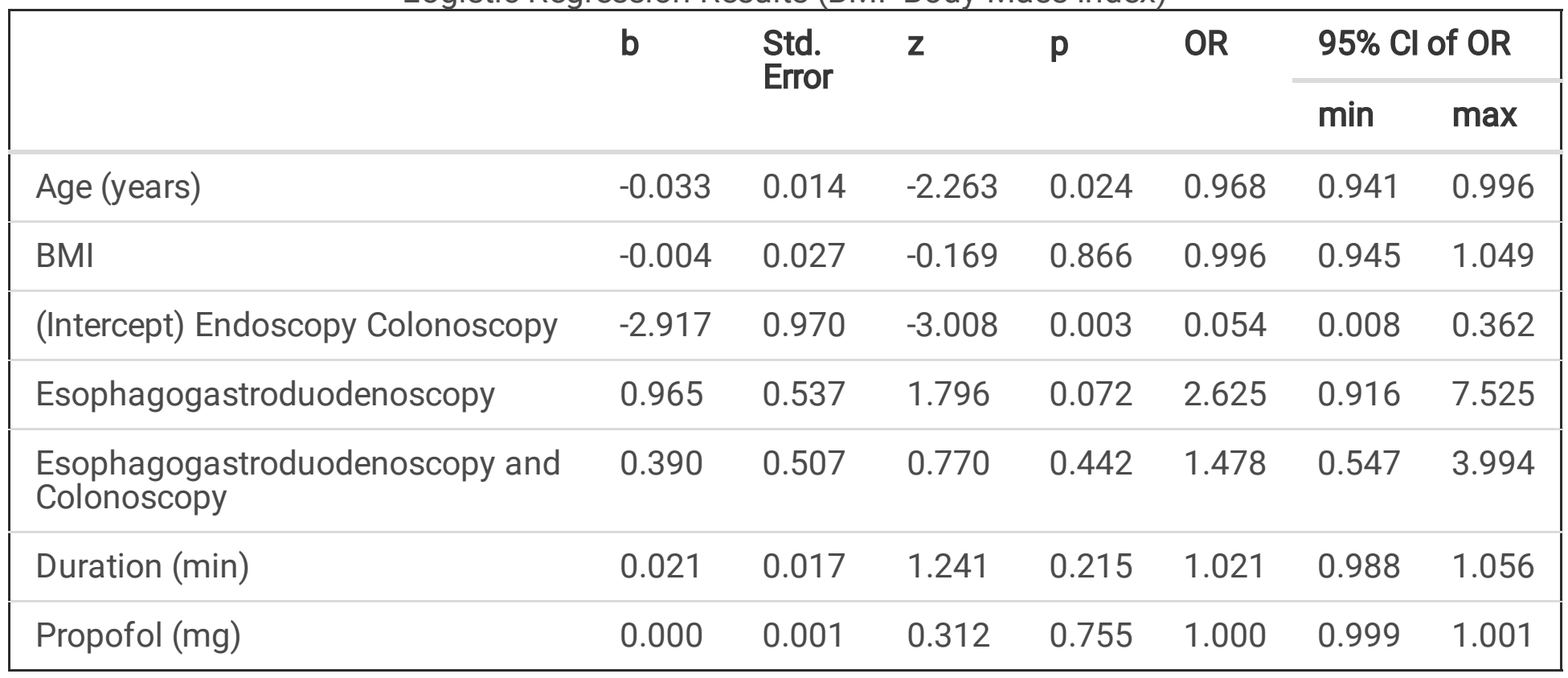

For patients with fever, the highest temperature measured was 39.8 degrees Celsius, with fever-like symptoms as chills, weakness, body and joint pain with shivering. Only one patient visited the emergency department due to the presence of fever and existing accompanying symptoms, while $80 \%$ of patients took antipyretic medications to alleviate the existing discomfort and temperature.

The most common single procedure performed was oesophagogastroduodenoscopy (41.2\%). Colonoscopy was performed as a single procedure in $17.6 \%$ and double (oesophagogastroduodenoscopy and colonoscopy) in $41.2 \%$ of our patients. Polypectomy was performed in only 5 patients $(14.7 \%)$. Multiple biopsies were performed in 26 of the 34 patients (76.5\%). Data analysis suggest that both oesophagogastroduodenoscopy and oesophagogastroduodenoscopy with colonoscopy have higher probability of fever relative to colonoscopy, but these relationships aren't statistically significant at the .05 level. 
Table 3

Data are presented as mean \pm SD for length of procedure and amount of Propofol that is used during procedure

\begin{tabular}{|c|c|c|c|c|}
\hline & & Fever & w/o Fever & $\mathbf{p}$ \\
\hline \multirow{3}{*}{$\begin{array}{l}\text { Length of } \\
\text { procedure } \\
\text { (min) }\end{array}$} & Esophagogastroduodenoscopy & $14.5 \pm 15.3$ & $9.1 \pm 8.5$ & .026 \\
\hline & Colonoscopy & $24 \pm 18.2$ & $21.4 \pm 10.8$ & .556 \\
\hline & $\begin{array}{l}\text { Esophagogastroduodenoscopy and } \\
\text { colonoscopy }\end{array}$ & $25.3 \pm 5.3$ & $28.2 \pm 10.3$ & .287 \\
\hline \multirow{3}{*}{$\begin{array}{l}\text { Propofol } \\
\text { mg }\end{array}$} & Esophagogastroduodenoscopy & $339.3 \pm 208.6$ & $271.2 \pm 130.2$ & .065 \\
\hline & Colonoscopy & $467 \pm 186$ & $438.4 \pm 406$ & .866 \\
\hline & $\begin{array}{l}\text { Esophagogastroduodenoscopy and } \\
\text { colonoscopy }\end{array}$ & $489 \pm 167.7$ & $489 \pm 271$ & .997 \\
\hline
\end{tabular}

Data recording length of the procedure and the total amount of propofol that was used during the procedure are presented in Table 3. Propofol was used in almost all of the endoscopy procedures in our institution. The dose was directly related to the duration of the procedure. Related to fever, both amount of propofol and duration of procedure aren't statistically significant at the .05 level.

Co-administered medications Fentanyl $(50-100 \mathrm{mcg})$ and Lidocaine $(50-100 \mathrm{mg})$ are very often used as a part of MAC protocol. Other medications like Glycopyrrolate, Ondansetron, or Metoclopramide were rarely used and based on our limited data it is not possible to determine a cause-effect relationship.

Co-morbidities related to patients with fever are presented in Table 4. The most common co-morbidity was hypertension (20.6\%) followed by diabetes mellitus (14.6\%).

Table 4

Co-morbidities, presented as absolute number (n) and percentage (\%)

\begin{tabular}{|ll|}
\hline Hypertension (HTA) & 7/34 (20.6\%) \\
\hline Diabetes Mellitus (DM) & $5 / 34(14.7 \%)$ \\
\hline Asthma & $3 / 34(8.8 \%)$ \\
\hline Hypothyroidism & $3 / 34(8.8 \%)$ \\
\hline Cirrhosis & $3 / 34(8.8 \%)$ \\
\hline Chronic Kidney Disease & $3 / 34(8.8 \%)$ \\
\hline Arthritis & $3 / 34(8.8 \%)$ \\
\hline Coronary artery Disease (CAD) & $2 / 34(5.9 \%)$ \\
\hline Cerebrovascular Accident (CVA) & $1 / 34(2.9 \%)$ \\
\hline
\end{tabular}

Page $7 / 13$ 
During the study, the authors also monitored the relationship between fever and the type of scope used during the procedure. Each case of fever was associated with a different scope, which excludes the possibility that the scope itself could be a potential source of infection and fever due to the existence of possible damage in the endoscope channel. Also, a cause-effect relationship between fever and a specific technique by the endoscopist or anaesthetist has not been observed.

Bearing in mind that the subject of the study were non-hospitalized patients and that we conducted a phone questionnaire that was conducted 24 hours after the endoscopic procedure, we were not able to perform laboratory analysis in patients positive for fever. Pro-inflammatory markers were not followed. Based on auto-anamnestic data in all patients, the temperature occurred in the first 24 hours in the form of a self-limiting episode with all of the accompanying symptoms, after which it completely resolved spontaneously or after a single dose of paracetamol. Repeated episodes of fever were not reported.

\section{Discussion}

Published literature on post-endoscopy fever is limited. Most studies that analysed post-endoscopic fever were analysed after polypectomy and in most cases only in hospitalised patients. Lee and colleagues [7] reported a case-control study of patients who experienced post-polypectomy fever (PPF), defined as elevated temperature after polypectomy without evidence of other explainable fever foci. Although their findings do not explain the possible mechanisms underlying PPF, risk factors are defined as hypertension and large polyps. In our study, we monitored PEF incidence in a non-hospitalised, predominantly healthy population in which screening endoscopic procedures were performed. Polypectomy was performed in only five PEF patients (14.7\%) and the most common single was oesophagogastroduodenoscopy (41.2\%).

The mechanisms of PEF are complex and it is not yet fully explained. While its outcome is generally favorable, PEF can generate patient discomfort, fear and unnecessary diagnostic procedures as well as possible hospitalisations.

Endogenous immune response has not been sufficiently examined for gastro-colonic endoscopic procedures, but it has been for bronchoscopy procedures [8]. Fever after bronchoscopy, especially bronchoalveolar lavage (BAL) suggested by several authors may initiate by an endogenous immune response. A systemic inflammatory response is characterized by an increase in circulating cytokine levels such as tumor necrosis factor (TNF)-alpha, interleukin (IL)-1 beta, and IL-6 [9-12]. During polypectomy, adenomatous polyps have an inflammatory stromal microenvironment, rich in macrophages, neutrophils, and $T$ helper cells [13] that can be a trigger for endogenous immune response.

Alternatively, endoscopic examinations may be the gateway through which bacteria invade the body. Transient bacteremia after colonoscopy, with or without polypectomy, occurs in approximately $4 \%$ of procedures, with a range of $0-25 \%[14,15]$ The reported incidence of bacteremia after diagnostic upper GI endoscopy, with or without biopsies, was less than $8 \%$ comparing bacteremia after colonoscopy, with or without biopsies and polypectomies, which ranges from $0-25 \%$ [16]. 
Post-polypectomy coagulation syndrome (PPCS) is an uncommon complication (incidence 0.003-0.1\%) after colonoscopy as described in the literature. This syndrome is the result of an electrocoagulation injury to the colonic wall that induces a transmural burn and localised peritoneal inflammation without evidence of perforation on radiographic studies. PPCS has typical symptoms such as abdominal pain, fever, leukocytosis and peritoneal tenderness [17]. Factors associated with an increased risk include large polyp size, non-polypoid shape of lesion, caecum or ascending colon location, and patient history of hypertension.

Contaminated endoscopes can also potentially be associated with endoscopy healthcare-associated infections and possible PEF. Flexible endoscopes may become heavily contaminated with blood, secretions and microorganisms during use. They are difficult to clean and disinfect, and easy to damage because of their complex design with narrow lumens and multiple internal channels. In addition, the ability of bacteria to form biofilms in the endoscope channels, especially when these become damaged, can contribute to the failure of the decontamination process $[16,18]$. In a study published by Gorse and Messner, the authors reported that endoscopy-related infections, usually bacterial, occurred at $6 \%$ of institutions that participated in a national survey [19]. Our data is inconsistent with the above study as we could not establish a causal relationship between fever and gastro or colonoscopes used in clinical practice.

Although high blood pressure was marked as contributing factor that promotes post polypectomy fever in a previously published study [7], the mechanism is still unclear. Possibly, blood pressure-regulatory systems, such as the renin-angiotensin system and sympathetic nervous system, interact with the proinflammatory cytokines, such as IL-6 and TNF-a as a possible potentional source of PEF [17, 20].

Propofol-induced drug fever during endoscopy is not new [21, 22], however it is not well known. In 2007, the U.S. Food and Drug Administration (FDA) released a safety alert concerning reports of cases of fever, chills, and body aches in patients shortly after the administration of propofol. New episodes of fever have been associated with patients following gastrointestinal procedures. Although the propofol emulsion is capable of supporting microbial growth in the event of contamination during administration, the tests performed by the FDA did not identify any units contaminated with bacteria or endotoxins [23]. Study published by Bennett and colleagues [24] investigated an unusual outbreak of bloodstream infections, surgical-site infections, and acute febrile episodes after surgical procedures between June 1990 and February 1993. Exposure to propofol was the only significant variable associated with postoperative complications. Although cultures of unopened containers of propofol were negative, cultures of propofol from syringes were positive at two hospitals. It was found that the contamination did not occur as inadequate sterility of propofol emulsion but to accidental extrinsic contamination. After this event, the product has been reformulated to contain disodium edetate $0.005 \%$, to inhibit the growth of microorganisms in the event of accidental contamination. However, propofol can still support the growth of microorganisms, as it is not an antimicrobial preserved product under the United States Pharmacopeia standards and aseptic precautions needs to be maintained during administration. 
Several limitations in this study should be noted. First, we cannot provide information on microbiological workup for patients who developed a post-endoscopy fever. The number of patients with PEF was too small to identify the risk factors for this condition. Finally, this study was performed as a single-center study, and a larger multicenter study would give better insight into this problem. Further investigation on these limitations is recommended.

\section{Conclusion}

Our findings do not fully explain the possible mechanisms underlying PEF but our study should increase awareness regarding PEF as a common side effect after endoscopy. PEF is an unpleasant event and it is associated with patient discomfort, dissatisfaction and fear during their post-endoscopy recovery, with the possibility of causing unnecessary investigations as well as possible hospitalizations.

\section{Declarations}

\section{Ethics approval and consent to participate}

Ethics approval for study was provided by the Institutional Review Board (IRB) of Cleveland Clinic, Abu Dhabi, UAE, under Study Registration No. A-2019-046, on 9th September 2019. For 34 patients with fever verbal telephone consent was obtained. Consent for the remaining 1020 patients with no fever for retrospective data analysis was waived by same study registration number (the IRB of Cleveland Clinic, Abu Dhabi, UAE, Study Registration No. A-2019-046, on 9th September 2019).

This study followed the "Strengthening the Reporting of Observational Studies in Epidemiology (STROBE)" statement guidelines for observational studies.

\section{Consent for publication}

Not applicable.

\section{Availability of data and materials}

The datasets generated and/or analysed during the current study are not publicly available due Cleveland Clinic Abu Dhabi, Institutional Review Board police, but are available from the corresponding author on reasonable request and with permission of Cleveland Clinic Abu Dhabi.

\section{Competing interests}

The authors declare no potential conflicts of interest with respect to the research, authorship and/or publication of this article.

\section{Funding}

The authors received no financial support for the authorship and publication of this article. 


\section{Authors' contributions}

Author contributions to the study and manuscript preparation include the following: Conception and design: Boris Tufegdzic; Drafting the article: Boris Tufegdzic; Critically revising the article: all authors; Reviewed submitted version of manuscript: all authors; Approved the final version of the manuscript on behalf of all authors: Boris Tufegdzic; Study supervision: Boris Tufegdzic

\section{Acknowledgements}

Authors acknowledge Dr Anthony $\mathrm{J}$ Cartwright for his contribution in grammar and spelling revision of the manuscript.

\section{References}

1. https://en.wikipedia.org/wiki/Fever

2. Samer Ammar, Pfefferkorn, Croffie JM, Gupta SK, Corkins MR, Fitzgerald JF. Complications after outpatient upper GI endoscopy in children: 30-day follow-up. Am J Gastroenterol. 2003 Jul;98(7):1508-11.

3. Kramer RE, Narkewicz MR. Adverse Events Following Gastrointestinal Endoscopy in Children: Classifications, Characterizations, and Implications. J Pediatr Gastroenterol Nutr. 2016 Jun;62(6):828-33. doi: 10.1097/MPG.0000000000001038. PMID: 26594830.

4. Boster JM, Iwanowski M, Kramer RE. Management of Pediatric Postendoscopy Fever: Reducing Unnecessary Health Care Utilization With a Clinical Care Guideline. J Pediatr Gastroenterol Nutr. 2021 Feb 1;72(2):250-254. doi: 10.1097/MPG.0000000000002936. PMID: 32925556; PMCID: PMC8256551.

5. Coser RB, Dalio MB, Martins LCP, et al. Colonoscopy complications: experience with 8968 consecutive patients in a single institution. Revista do Colegio Brasileiro de Cirurgioes. 2018 Sep;45(4):e1858. DOI: 10.1590/0100-6991e-20181858. PMID: 30231113.

6. https://www.strobe-statement.org/checklists/

7. Lee SH, Kim KJ, Yang DH, et al. Postpolypectomy Fever, a rare adverse event of polypectomy: nested case-control study. Clin Endosc. 2014;47(3):236-241. doi:10.5946/ce.2014.47.3.236

8. Julia Kovaleva,Frans T. M. Peters, Henny C. van der Mei, John E. Degener: Transmission of Infection by Flexible Gastrointestinal Endoscopy and Bronchoscopy Clin Microbiol Rev. 2013 Apr; 26(2): 231254. doi: $10.1128 /$ CMR.00085-12

9. Terashima T, Amakawa K, Matsumaru A, van Eeden S, Hogg JC, Yamaguchi K. BAL induces an increase in peripheral blood neutrophils and cytokine levels in healthy volunteers and patients with pneumonia. Chest. 2001;119(6):1724-1729. doi:10.1378/chest.119.6.1724

10. Hackner K, Riegler W, Handzhiev S, et al. Fever after bronchoscopy: serum procalcitonin enables early diagnosis of post-interventional bacterial infection. BMC Pulm Med. 2017;17(1):156. Published 2017 Nov 28. doi:10.1186/s12890-017-0508-1 
11. Nelson ME, Wald TC, Bailey K, Wesselius LJ. Intrapulmonary cytokine accumulation following BAL and the role of endotoxin contamination. Chest. 1999;115(1):151-157. doi:10.1378/chest.115.1.151

12. Standiford TJ, Kunkel SL, Strieter RM. Elevated serum levels of tumor necrosis factor-alpha after bronchoscopy and bronchoalveolar lavage. Chest.1991;99(6):1529-30.

13. McLean MH, Murray GI, Stewart KN, et al. The inflammatory microenvironment in colorectal neoplasia. PLoS One. 2011;6(1):e15366. Published 2011 Jan 7. doi:10.1371/ journal.pone.0015366

14. Low DE, Shoenut JP, Kennedy JK, et al. Prospective assessment of risk of bacteremia with colonoscopy and polypectomy. Dig Dis Sci. 1987;32(11):1239-1243. doi:10.1007/BF01296372

15. Botoman VA, Surawicz CM. Bacteremia with gastrointestinal endoscopic procedures. Gastrointest Endosc. 1986;32(5):342-346. doi:10.1016/s0016-5107(86)71880-4

16. Kovaleva J, Peters FT, van der Mei HC, Degener JE. Transmission of infection by flexible gastrointestinal endoscopy and bronchoscopy. Clin Microbiol Rev. 2013;26(2):231-254. doi:10.1128/CMR.00085-12

17. Cha JM, Lim KS, Lee SH, et al. Clinical outcomes and risk factors of post-polypectomy coagulation syndrome: a multicenter, retrospective, case-control study. Endoscopy. 2013;45(3):202-207. doi:10.1055/s-0032-1326104

18. Buss AJ, Been MH, Borgers RP, et al. Endoscope disinfection and its pitfalls-requirement for retrograde surveillance cultures. Endoscopy. 2008;40(4):327-332. doi:10.1055/s-2007-995477

19. Gorse GJ, Messner RL. Infection control practices in gastrointestinal endoscopy in the United States: a national survey. Gastroenterol Nurs. 1991;14(2):72-79. doi:10.1097/00001610-199110000-00003

20. Granger JP. An emerging role for inflammatory cytokines in hypertension. Am J Physiol Heart Circ Physiol. 2006;290(3):H923-H924. doi:10.1152/ajpheart.01278.2005

21. Yatabe T, Yamashita K, Yokoyama M.: Drug fever caused by propofol in the intensive care unit. $J$ Anesth. 2015 Oct;29(5):786-9. doi: 10.1007/s00540-015-2007-y. Epub 2015 Mar 24.

22. Blossom DB, Chen TH, Li J, Langer AJ, Carpenter LR, Glenshaw MT, Gould CV, Weltman A, Srinivasan A.: Self-limited febrile syndromes temporally associated with the use of propofol for sedation in gastrointestinal endoscopic procedures.

23. FDA alert/information for healthcare professionals: propofol (marketed as Diprivan and as generic products) Information. U.S. Food and Drug Administration, Center for Drug Evaluation and Research. Available at: https://www.fda.gov/drugs/postmarket-drug-safety-information-patients-andproviders/propofol-marketed-diprivan-and-generic-products-information

24. Bennett SN, McNeil MM, Bland LA, et al. Postoperative infections traced to contamination of an intravenous anesthetic, propofol. N Engl J Med. 1995;333(3):147-154. doi:10.1056/NEJM199507203330303

\section{Figures}




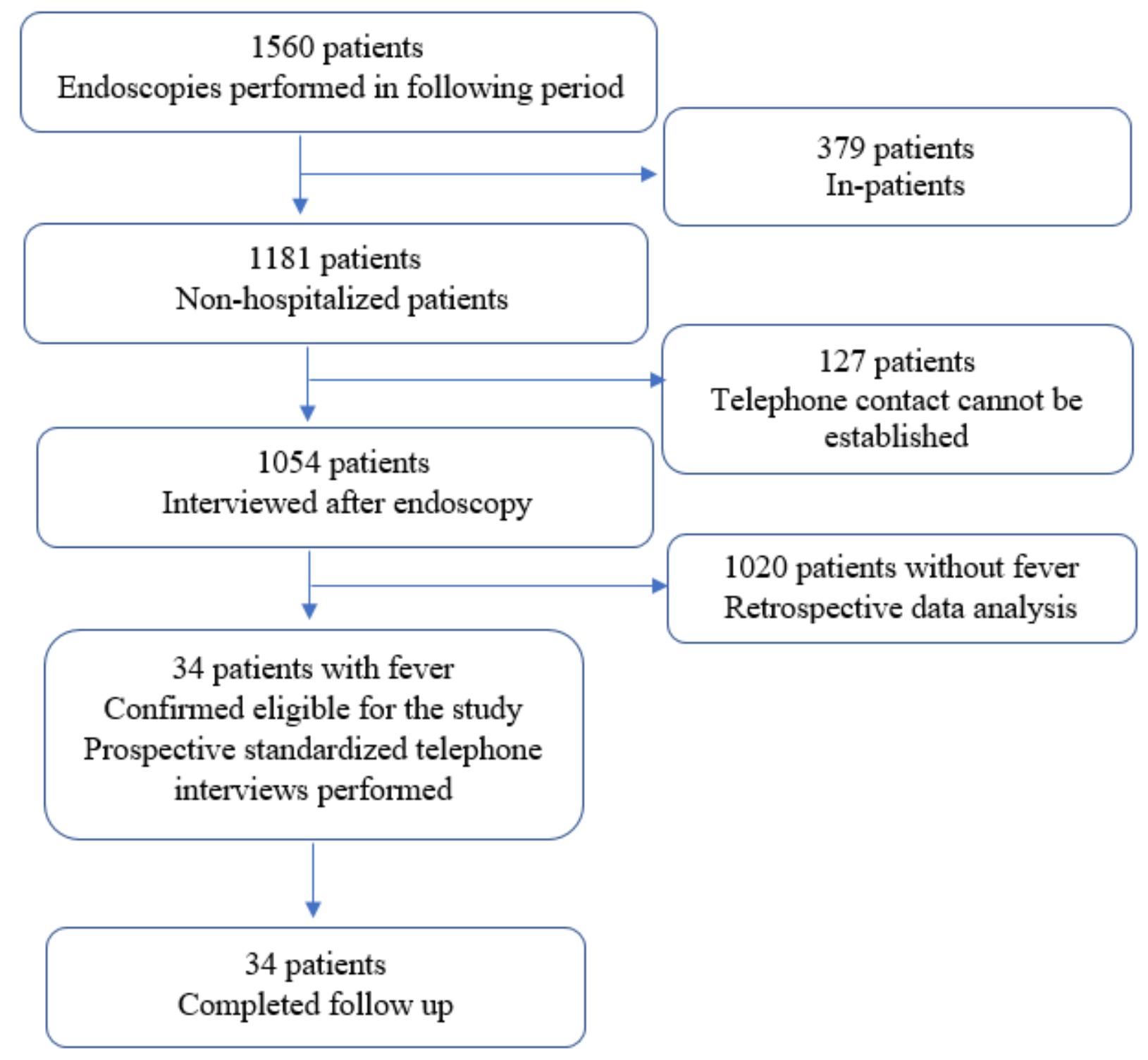

Figure 1

Flow chart of study process 